\title{
Persistent N2 disease after neoadjuvant chemotherapy for non-small-cell lung cancer
}

\author{
Kristin A. Higgins, MD, ${ }^{\mathrm{a}}$ Junzo P. Chino, MD, ${ }^{\mathrm{a}}$ Neal Ready, MD, ${ }^{\mathrm{b}}$ Mark W. Onaitis, MD, ${ }^{\mathrm{c}}$ \\ Mark F. Berry, MD, ${ }^{\mathrm{c}}$ Thomas A. D’Amico, MD, ${ }^{\mathrm{c}}$ and Chris R. Kelsey, MD ${ }^{\mathrm{a}}$
}

\begin{abstract}
Objectives: Patients achieving a mediastinal pathologic complete response with neoadjuvant chemotherapy have improved outcomes compared with patients with persistent $\mathrm{N} 2$ disease. How to best manage this latter group of patients is unknown, prompting a review of our institutional experience.
\end{abstract}

\begin{abstract}
Methods: All patients who initiated neoadjuvant therapy for non-small-cell lung cancer from 1995 to 2008 were evaluated. The patients were excluded if they had received preoperative radiotherapy, had had a mediastinal pathologic complete response, or had evidence of disease progression after neoadjuvant chemotherapy. The clinical endpoints were calculated using the Kaplan-Meier product-limit method and compared using a log-rank test.
\end{abstract}

Results: A total of 28 patients were identified. The median follow-up period was 24 months. Several neoadjuvant chemotherapy regimens were used, most commonly carboplatin with vinorelbine $(36 \%)$ or paclitaxel $(32 \%)$. A partial response to chemotherapy was noted in $23(82 \%)$ and stable disease was noted in $5(18 \%)$ on postchemotherapy imaging. Resection was performed in 22 of 28 patients, consisting of lobectomy in 14 , pneumonectomy in 2 , and wedge/segmentectomy in $6(21 / 22 \mathrm{R} 0,1 / 22 \mathrm{R} 1)$. There were no postoperative deaths. Postoperative therapy (radiotherapy and/or additional chemotherapy) was administered to 12 patients (55\%). The remaining 6 patients generally received definitive radiotherapy with or without additional chemotherapy. The overall and disease-free survival rate at 1,3 , and 5 years was $75 \%, 37 \%$, and $37 \%$ and $50 \%, 23 \%$, and $19 \%$, respectively. The survival rate at 5 years was similar between patients undergoing resection $(34 \%)$ and those receiving definitive radiotherapy with or without chemotherapy $(40 \% ; P=.73)$.

Conclusions: Disease-free and overall survival was sufficiently high to warrant aggressive local therapy (surgery or radiotherapy) in patients with persistent N2 disease after neoadjuvant chemotherapy. (J Thorac Cardiovasc Surg 2011;142:1175-9)

The optimal treatment approach for patients with operable stage III (N2) non-small-cell lung cancer (NSCLC) is controversial. Upfront surgery for patients with clinically apparent N2 disease is unsatisfactory, with long-term survival rates less than $10 \% .{ }^{1}$ However, a surgical approach, incorporating neoadjuvant chemotherapy, is often used for healthy patients with a minimal $\mathrm{N} 2$ disease burden.

Approximately $17 \%$ to $53 \%$ of patients achieve a mediastinal pathologic complete response with chemotherapy alone. ${ }^{2-9}$ Several studies have shown that patients who clear their mediastinal lymph nodes with neoadjuvant chemotherapy have improved outcomes compared with patients with

\footnotetext{
From the Department of Radiation Oncology, ${ }^{a}$ Division of Medical Oncology, ${ }^{b}$ Department of Medicine, and Division of Cardiovascular and Thoracic Surgery, ${ }^{c}$ Department of Surgery, Duke University Medical Center, Durham, NC.

Disclosures: Authors have nothing to disclose with regard to commercial support.

Presented at the 14th World Conference on Lung Cancer, July 3-7, 2011, Amsterdam, The Netherlands.

Received for publication April 16, 2011; revisions received July 11, 2011; accepted for publication July 26, 2011.

Address for reprints: Kristin A. Higgins, MD, Emory Department of Radiation Oncology, 1365 Clifton Rd NE, Atlanta, GA 30322 (E-mail: kristin.higgins@ emory.edu).

$0022-5223 / \$ 36.00$

Copyright $(C 2011$ Published by Elsevier Inc. on behalf of The American Association for Thoracic Surgery

doi:10.1016/j.jtcvs.2011.07.059
}

persistent N2 disease. ${ }^{2,8-13}$ For example, in a prospective Phase II study evaluating neoadjuvant cisplatin and docetaxel, pathologically negative mediastinal lymph nodes after chemotherapy were associated with a significantly improved 3-year survival rate compared with that for patients with persistent $\mathrm{N} 2$ disease $(61 \% \text { vs } 11 \%)^{2}$

Although persistent N2 disease after neoadjuvant chemotherapy can be associated with inferior outcomes, the optimal approach for these patients remains unclear. Specifically, whether surgery should proceed despite an incomplete pathologic response or whether a definitive course of radiotherapy, with or without additional chemotherapy, should be pursued is not known. We explored this further by evaluating our own experience at the Duke University Medical Center.

\section{METHODS}

This retrospective analysis was approved by the Duke University Medical Center institutional review board. All patients with NSCLC who were treated with neoadjuvant chemotherapy before planned surgical resection were identified from the Duke Comprehensive Cancer Center's registry. Those patients with pathologically confirmed persistent N2 disease after chemotherapy alone but no evidence of local or distant disease progression were identified. Patients who were pNO-N1 after chemotherapy or had evidence of local or distant disease progression were therefore excluded. Any patients who received preoperative radiotherapy, including those 


\section{Abbreviations and Acronyms}

$\mathrm{CT}=$ computed tomography

NSCLC $=$ non-small-cell lung cancer

PET $=$ positron emission tomography

with superior sulcus tumors, were excluded. The medical records and pertinent radiologic imaging studies were reviewed to obtain the clinical, surgical, and pathologic characteristics of each patient.

All patients underwent comprehensive staging studies initially to establish the extent of disease, including history and physical examination, imaging, and routine blood work. Pulmonary function tests were routinely obtained, either during the initial workup or immediately before surgery. After evaluation by a multidisciplinary team, including a thoracic surgeon, medical oncologist, and radiation oncologist, neoadjuvant chemotherapy was initiated. Because this was not standardized, a variety of regimens were used.

Computed tomography (CT) or positron emission tomography (PET)/ CT imaging was obtained in all patients after they completed neoadjuvant chemotherapy. In the absence of progressive disease, the patients then underwent additional workup, including mediastinoscopy, video-assisted thoracoscopic surgery, and/or thoracotomy, to assess the response to therapy and evaluate for possible disease progression before the planned lung resection. Based on the intraoperative assessment, resection proceeded at the discretion of the thoracic surgeon. Additional therapy after surgery, specifically radiotherapy and/or additional chemotherapy, was recommended at the discretion of the multidisciplinary team.

\section{Statistical Analysis}

Overall survival, disease-free survival, and local control were estimated using the Kaplan-Meier product-limit method. ${ }^{14}$ Overall survival was calculated from the date of diagnosis to the date of death. Patients were censored at the time of last follow-up. Disease-free survival was determined from the date of diagnosis to the date of the first failure or death. Local failures were cumulatively scored (ie, patients were not censored for local failure at the point of distant failure). Comparisons in clinical outcomes between patient subgroups were made using the log-rank test. ${ }^{15}$

\section{RESULTS}

Between 1995 and 2008, 301 patients were identified who received neoadjuvant chemotherapy for NSCLC at the Duke University Medical Center. Among these were 227 patients who also received neoadjuvant radiotherapy, including patients with superior sulcus tumors $(n=30)$, who were excluded. Of the remaining 74 patients, 35 were pathologically N0-N1 and 7 had evidence of local or distant disease progression by imaging or intraoperative findings after neoadjuvant chemotherapy. Four additional patients were excluded because they had received neoadjuvant therapy with single-agent dasatinib on a clinical trial $(\mathrm{n}=2)$, had known distant disease at diagnosis $(\mathrm{n}=1)$, or had received neoadjuvant chemotherapy at Duke but had undergone surgery elsewhere $(n=1)$. This left 28 patients with confirmed persistent N2 nodal positivity after neoadjuvant chemotherapy without evidence of disease progression. The median follow-up was 22 months for all patients and 92 months for the survivors.
TABLE 1. Patient and treatment characteristics

\begin{tabular}{|c|c|}
\hline Characteristic & Value \\
\hline \multicolumn{2}{|l|}{ Age, y } \\
\hline Median & 60 \\
\hline Range & $42-74$ \\
\hline \multicolumn{2}{|l|}{ Race } \\
\hline African-American & $4(14)$ \\
\hline Caucasian & $23(82)$ \\
\hline Other & $1(4)$ \\
\hline \multicolumn{2}{|l|}{ Gender } \\
\hline Male & $17(61)$ \\
\hline Female & $11(39)$ \\
\hline \multicolumn{2}{|l|}{ Clinical T stage* } \\
\hline T1a & $5(18)$ \\
\hline $\mathrm{T} 1 \mathrm{~b}$ & $6(21)$ \\
\hline $\mathrm{T} 2 \mathrm{a}$ & $6(21)$ \\
\hline $\mathrm{T} 2 \mathrm{~b}$ & $2(7)$ \\
\hline $\mathrm{T} 3$ & $5(18)$ \\
\hline $\mathrm{T} 4$ & $1(4)$ \\
\hline Unknown & $3(11)$ \\
\hline \multicolumn{2}{|c|}{ Involved N2 lymph node stations $\dagger$} \\
\hline 1 Station & $17(61)$ \\
\hline 2 Stations & $6(21)$ \\
\hline No sampling & $5(18)$ \\
\hline \multicolumn{2}{|l|}{ Neoadjuvant chemotherapy } \\
\hline Carboplatin/second agent $\ddagger$ & $19(68)$ \\
\hline Cisplatin/second agent $\ddagger$ & $6(21)$ \\
\hline Docetaxel & $3(11)$ \\
\hline \multicolumn{2}{|l|}{ Surgery } \\
\hline Wedge/segmentectomy & $6(21)$ \\
\hline Lobectomy & $14(50)$ \\
\hline Pneumonectomy & $2(7)$ \\
\hline None & $6(21)$ \\
\hline
\end{tabular}

Data in parentheses are percentages. $*$ TNM 7 . $\nmid$ Per preoperative mediastinoscopy. $\ddagger$ Vinorelbine, paclitaxel, docetaxel, or pemetrexed.

Initial staging consisted of PET/CT $(n=18,64 \%)$ or CT $(\mathrm{n}=10,36 \%)$. The median forced expiratory volume in 1 second and carbon monoxide diffusing capacity was $83 \%$ (range, $42 \%-113 \%$ ) and $75 \%$ (range, $46 \%-108 \%$ ), respectively. All patients initiated neoadjuvant chemotherapy for stage IIIA (N2) NSCLC. Mediastinal lymph nodes were pathologically confirmed in $23(82 \%)$ and presumed positive by imaging studies in 5 patients $(18 \%)$. The patient and treatment characteristics can be found in Table 1. In general, this cohort of patients had fairly small primary tumors with a median size of $3 \mathrm{~cm}$. Similarly, the N2 disease burden was minimal, with a median size of the largest $\mathrm{N} 2$ node of $1.5 \mathrm{~cm}$ (range, $<1-3$ ) and a median number of radiographically involved $\mathrm{N} 2$ nodal stations of 1 (range, $0-3)$. Of the patients undergoing initial mediastinscopy, the median number of pathologically involved N2 nodal stations was 1 (range, 1-2).

A variety of chemotherapy regimens was used, primarily carboplatin combined with vinorelbine or paclitaxel 
Persistent N2 after Neoadjuvant Chemotherapy

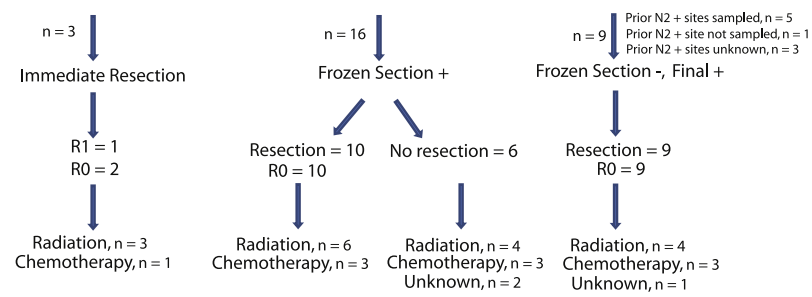

FIGURE 1. Outline of patients and treatment administered after neoadjuvant chemotherapy.

(Table 1). The median number of cycles was 2 (range, 2-4). After completing neoadjuvant chemotherapy, imaging was repeated in all patients (CT in $86 \%$ and PET/CT in 14\%). A response to chemotherapy was noted in $23(82 \%)$, and stable disease was noted in $5(18 \%)$, with no patients achieving a complete response by imaging.

Figure 1 details the subsequent treatment for all 28 patients and provides pathologic data regarding the frozen section results of initial intraoperative N2 sampling, whether all previously documented pathologically involved $\mathrm{N} 2$ lymph node stations were sampled, and whether a R0 resection was performed. In brief, resection was performed in 22 of 28 patients and consisted of lobectomy in 14, pneumonectomy in 2, and wedge/segmentectomy in 6 . An R0 resection was achieved in 21 of 22 patients, with 1 patient receiving $\mathrm{R} 1$ resection because of a positive surgical margin after chest wall resection. The median number of pathologically positive mediastinal lymph node stations after resection was 1 . No postoperative deaths occurred. Of the 22 patients undergoing lung resection, $13(59 \%)$ received postoperative RT and $7(32 \%)$ received additional chemotherapy postoperatively. One patient was lost to follow-up, and it was unknown whether they had received any adjuvant therapy.

The remaining 6 patients did not undergo resection. The stated reason for all patients was persistent N2 disease detected during mediastinoscopy or video-assisted thoracic surgery exploration. Of these, 4 patients received radiotherapy (dose range, 60-70 Gy) to definitive doses, 3 of whom received additional concurrent chemotherapy. Two patients were referred for definitive radiotherapy at outside institutions but the receipt of such could not be documented.

The median survival for all patients was 22 months. The overall and disease-free survival rate at 1,3 , and 5 years was $75 \%, 37 \%$, and $37 \%$ and $50 \%, 23 \%$, and $19 \%$, respectively (Figure 2). The local disease control rate at 1, 3, and 5 years was $73 \%, 53 \%$, and $53 \%$, respectively.

The overall survival rate at 5 years was similar between patients undergoing resection $(34 \%)$ and those receiving definitive radiotherapy with or without chemotherapy $(40 \% ; P=.73)$, as demonstrated in Figure 3 . At 5 years, the overall survival rate $(52 \%$ vs $30 \%, P=.23)$, disease-

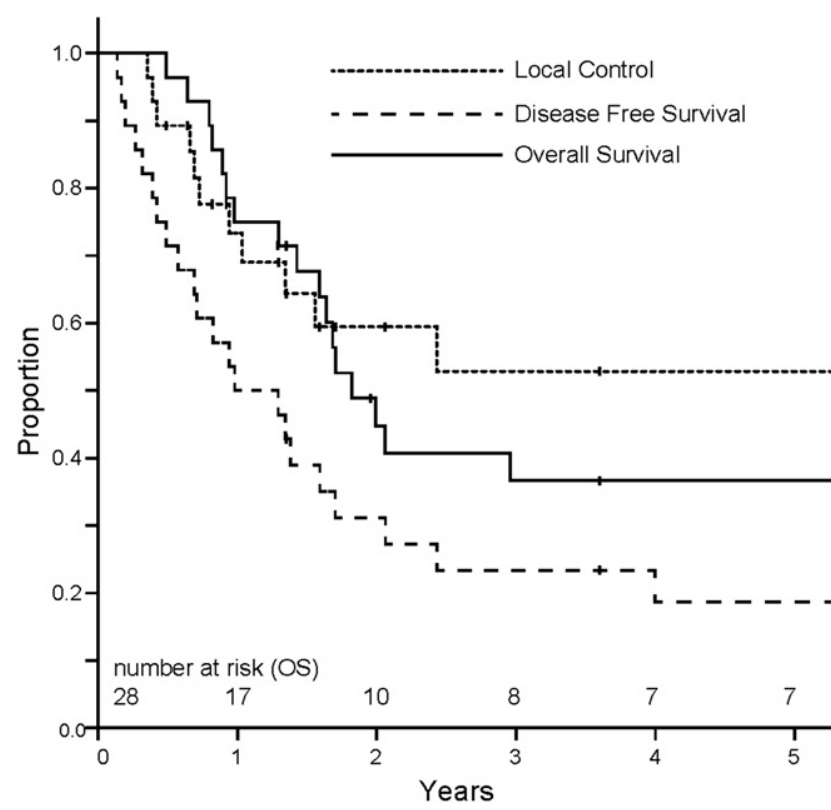

FIGURE 2. Kaplan-Meier curve demonstrating overall survival (OS), disease-free survival, and local control for entire cohort of patients with persistent N2 disease after neoadjuvant chemotherapy.

free survival rate $(28 \%$ vs $10 \%, P=.1)$, and local control rate ( $76 \%$ vs $51 \%, P=.40$ ) were not significantly different statistically between those patients with negative frozen section mediastinal lymph node sampling and those patients with positive frozen section mediastinal lymph node sampling, although the numbers in each group were small.

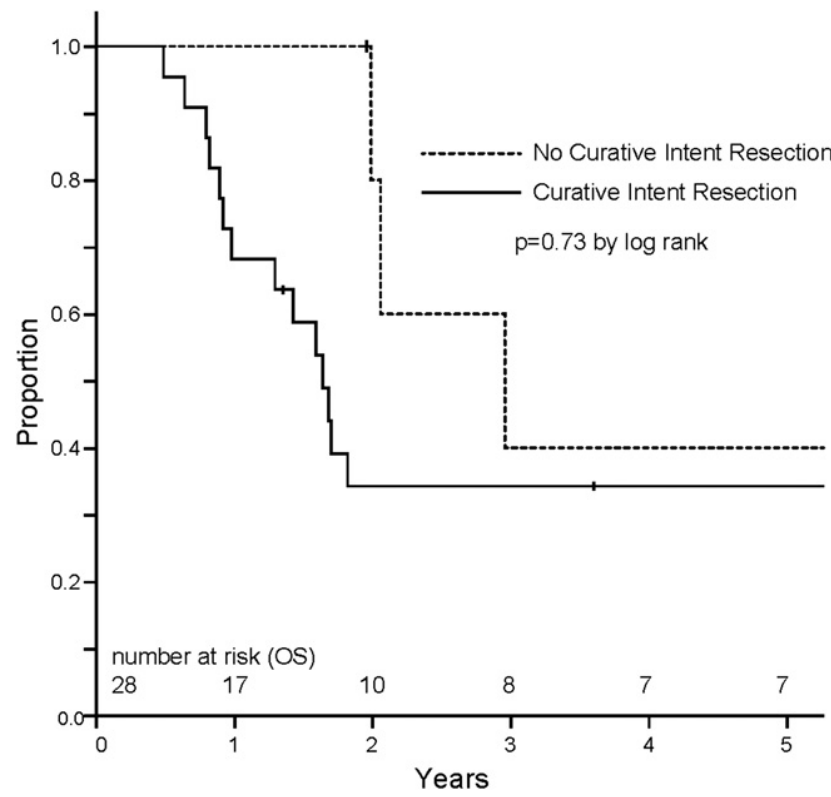

FIGURE 3. Kaplan-Meier curve demonstrating overall survival $(O S)$ for patients with persistent $\mathrm{N} 2$ disease undergoing resection versus nonoperative treatment. 


\section{DISCUSSION}

The optimal approach for resectable N2 NSCLC remains controversial. Neoadjuvant chemotherapy has been shown to improve survival relative to surgery alone in some, ${ }^{4,16,17}$ but not all, ${ }^{3,18}$ randomized trials. Several prospective studies have shown that a mediastinal pathologic complete response is associated with improved outcomes after surgery compared with patients with residual N2 disease.,10-12 In fact, most studies have shown that long-term survival is less than $10 \%$ to $15 \%$ when patients remain $\mathrm{N} 2$ positive after neoadjuvant chemotherapy, prompting some to question the role of surgery in this group of patients. ${ }^{2,11,12}$ However, appropriate adjuvant therapies, including radiotherapy and additional chemotherapy, might be associated with better outcomes in these patients.

Although neoadjuvant chemoradiotherapy is also widely used for patients with $\mathrm{N} 2$ disease, this report focuses on patients treated with preoperative chemotherapy, because this is the preferred approach at our institution owing to the fewer postoperative complications ${ }^{19}$ and the ability to proceed with an uninterrupted course of radiotherapy if resection is not ultimately performed.

In the present study, patients with disease progression during neoadjuvant chemotherapy were excluded from the analysis. This was a relatively small number of patients $(n=7)$, and most studies have shown that disease progression during chemotherapy is relatively uncommon. ${ }^{6,16,17}$

Most patients in our study with persistent N2 disease after neoadjuvant chemotherapy proceeded to surgical resection $(79 \%)$. Many received postoperative radiotherapy and/or additional chemotherapy. Those patients who did not undergo resection generally received definitive radiotherapy ( $\geq 60 \mathrm{~Gy}$ ), many with concurrent chemotherapy. With this approach, the actuarial overall survival rate at 5 years was $37 \%$, demonstrating that in appropriately selected patients, persistent disease after neoadjuvant chemotherapy does not necessarily carry a grim prognosis and perhaps aggressive local therapy is warranted.

These somewhat unexpected high survival rates could be due to several reasons. First, although the number of patients was small, those patients with progression during chemotherapy were excluded. Second, the burden of N2 disease in our series was small, with a median number of involved $\mathrm{N} 2$ lymph nodes of 1 and a median size of $1.5 \mathrm{~cm}$. However, patients with bulky or multistation N2 disease are generally not considered optimal candidates for surgery at our institution, and most would not have received neoadjuvant chemotherapy. Third, the patients considered for surgery are generally younger, with a better performance status, than many patients with stage IIIA disease treated with a nonsurgical approach. Finally, patients received an aggressive, often trimodality approach, which might have decreased the risk of disease recurrence.
Our group has previously published a comparison of patients receiving neoadjuvant chemotherapy versus chemoradiotherapy for IIIA (N2) NSCLC. ${ }^{20}$ For the subgroup of patients $(\mathrm{n}=10)$ who achieved a mediastinal pathologic complete response to neoadjuvant chemotherapy, the 3 -year overall survival rate was $40 \%$. This compares with $37 \%$ for patients with persistent $\mathrm{N} 2$ disease in our analysis. Other published data of contemporary phase II and III studies have demonstrated a 3-year overall survival rate of $61 \%^{2}$ and a 5 -year overall survival rate ranging from $29 \%$ to $44 \%{ }^{11,21}$ after preoperative chemotherapy for patients achieving nodal downstaging to N0 or N1. Although overall survival rates of this magnitude were not reached in the present study, the outcomes were much better than expected for a subgroup of patients who did not respond favorably to chemotherapy.

Our study was limited by the small numbers and could not address an important question regarding the optimal local treatment modality for patients with persistent N2 disease. It appears that both surgery and definitive radiotherapy can provide reasonable local control and survival. However, only a few patients in our series received definitive radiotherapy. Our experience suggests that local therapy (surgery and/or radiotherapy) provides some benefit and should be considered in this population of patients. A definitive course of radiotherapy is generally recommended if resection is not performed ( $\geq 60 \mathrm{~Gy}$ ), ideally with concurrent chemotherapy.

We acknowledge other weaknesses and potential biases inherent in this retrospective study. Not all patients underwent PET imaging at the initial diagnosis. However, the outcomes were expected to be better owing to stage migration when more sensitive imaging modalities are used in the staging workup. Furthermore, not all patients had upfront pathologic staging of the mediastinum. That said, all patients had pathologic confirmation of persistent $\mathrm{N} 2$ disease after completing neoadjuvant chemotherapy. Finally, it is acknowledged that this represents a select group of patients still thought to be appropriate surgical candidates despite harboring residual mediastinal disease. Whether similar results would be achieved with a broader N2 population is not known.

It should be noted that our general approach after neoadjuvant chemotherapy is to reserve resection for patients with no evidence of residual mediastinal disease or at most single-station, microscopic persistent $\mathrm{N} 2$ disease that would not necessitate pneumonectomy. We generally use neoadjuvant chemotherapy alone, instead of chemoradiotherapy, thus allowing an uninterrupted course of definitive radiotherapy if resection is not performed.

Our study was limited by the small numbers; thus, additional hypothesis-generating subgroup analyses were not possible. As mentioned, no differences in outcomes were 
seen in the patients receiving surgery versus definitive radiotherapy. It was not possible to assess other important issues, such as the type of surgery, role of postoperative radiotherapy, optimal dose of radiotherapy, importance of additional chemotherapy, and so forth. However, it should be noted that most patients were treated with a comprehensive approach aimed to control both local and distant disease. A comparison of the clinical outcomes between patients with negative versus positive mediastinal sampling before lung resection demonstrated no statistically significant differences in the clinical outcomes, although clear trends were seen in favor of those with negative findings on frozen section analysis, suggesting that the overall N2 disease burden is likely prognostic.

\section{CONCLUSIONS}

With appropriate adjuvant therapy, local modalities should be considered in patients with persistent N2 disease after neoadjuvant chemotherapy.

\section{References}

1. Martini N, Flehinger BJ. The role of surgery in N2 lung cancer. Surg Clin North Am. 1987;67:1037-49.

2. Betticher DC, Hsu Schmitz SF, Totsch M, Hansen E, Joss C, von Briel C, et al. Mediastinal lymph node clearance after docetaxel-cisplatin neoadjuvant chemotherapy is prognostic of survival in patients with stage IIIA pN2 non-small-cell lung cancer: a multicenter phase II trial. J Clin Oncol. 2003;21:1752-9.

3. Depierre A, Milleron B, Moro-Sibilot D, Chevret S, Quoix E, Lebeau B, et al. Preoperative chemotherapy followed by surgery compared with primary surgery in resectable stage I (except T1N0), II, and IIIa non-small-cell lung cancer. J Clin Oncol. 2002;20:247-53.

4. Pass HI, Pogrebniak HW, Steinberg SM, Mulshine J, Minna J. Randomized trial of neoadjuvant therapy for lung cancer: interim analysis. Ann Thorac Surg. 1992; 53:992-8.

5. O'Brien ME, Splinter T, Smit EF, Biesma B, Krzakowski M, Tjan-Heijnen VC, et al. Carboplatin and paclitaxol (Taxol) as an induction regimen for patients with biopsy-proven stage IIIA N2 non-small cell lung cancer: an EORTC phase II study (EORTC 08958). Eur J Cancer. 2003;39:1416-22.

6. Betticher DC, Hsu Schmitz SF, Totsch M, Hansen E, Joss C, von Briel C, et al. Prognostic factors affecting long-term outcomes in patients with resected stage IIIA pN2 non-small-cell lung cancer: 5-year follow-up of a phase II study. Br J Cancer. 2006;94:1099-106.

7. Kirn DH, Lynch TJ, Mentzer SJ, Lee TH, Strauss GM, Elias AD, et al. Multimodality therapy of patients with stage IIIA, N2 non-small-cell lung cancer: impact of preoperative chemotherapy on resectability and downstaging. $J$ Thorac Cardiovasc Surg. 1993;106:696-702.

8. Paul S, Mirza F, Port JL, Lee PC, Stiles BM, Kansler AL, et al. Survival of patients with clinical stage IIIA non-small cell lung cancer after induction therapy: age, mediastinal downstaging, and extent of pulmonary resection as independent predictors. J Thorac Cardiovasc Surg. 2011;141:48-58.

9. Stefani A, Alifano M, Bobbio A, Grigoroiu M, Jouni R, Magdeleinat P, et al. Which patients should be operated on after induction chemotherapy for N2 non-small cell lung cancer? Analysis of a 7-year experience in 175 patients. J Thorac Cardiovasc Surg. 2010;140:356-63.

10. Garrido P, Gonzalez-Larriba JL, Insa A, Provencio M, Torres A, Isla D, et al Long-term survival associated with complete resection after induction chemotherapy in stage IIIA (N2) and IIIB (T4N0-1) non small-cell lung cancer patients: the Spanish Lung Cancer Group Trial 9901. J Clin Oncol. 2007;25: 4736-42.

11. Lorent N, De Leyn P, Lievens Y, Verbeken E, Nackaerts K, Dooms C, et al. Longterm survival of surgically staged IIIA-N2 non-small-cell lung cancer treated with surgical combined modality approach: analysis of a 7-year prospective experience. Ann Oncol. 2004:15:1645-53.

12. Bueno R, Richards WG, Swanson SJ, Jaklitsch MT, Lukanich JM, Mentzer SJ, et al. Nodal stage after induction therapy for stage IIIA lung cancer determines patient survival. Ann Thorac Surg. 2000;70:1826-31.

13. Eberhardt WE, Albain KS, Pass H, Putnam JB, Gregor A, Assamura H, et al. Induction treatment before surgery for non-small cell lung cancer. Lung Cancer. 2003;42(Suppl 1):S9-14.

14. Kaplan EM, Meier P. Non-parametric estimation from incomplete observations. J Am Stat Assoc. 1958;53:457-81

15. Mantel N. Evaluation of survival data and two new rank order statistics arising in its consideration. Cancer Chemother Rep. 1966;50:163-70.

16. Rosell R, Gomez-Codina J, Camps C, Maestre J, Padille J, Canto A, et al. A randomized trial comparing preoperative chemotherapy plus surgery with surgery alone in patients with non-small-cell lung cancer. $N$ Engl J Med. 1994;330: 153-8.

17. Roth JA, Fossella F, Komaki R, Ryan MB, Putnam JB Jr, Lee JS, et al. A randomized trial comparing perioperative chemotherapy and surgery with surgery alone in resectable stage IIIA non-small-cell lung cancer. J Natl Cancer Inst. 1994;86: 673-80.

18. Pisters KM, Vallieres E, Crowley JJ, Franklin WA, Bunn PA Jr, Ginsberg RJ, et al Surgery with or without preoperative paclitaxel and carboplatin in early-stage non-small-cell lung cancer: Southwest Oncology Group Trial S9900, an intergroup, randomized, phase III trial. J Clin Oncol. 2010;28:1843-9.

19. Thomas M, Rube C, Hoffknecht P, Macha HN, Freitag L, Linder A, et al. Effect of preoperative chemoradiation in addition to preoperative chemotherapy: a randomised trial in stage III non-small-cell lung cancer. Lancet Oncol. 2008;9: 636-48.

20. Higgins K, Chino JP, Marks LB, Ready N, D’Amico TA, Clough RW, et al. Preoperative chemotherapy versus preoperative chemoradiotherapy for stage III (N2) non-small-cell lung cancer. Int J Radiat Oncol Biol Phys. 2009;75:1462-7.

21. van Meerbeeck JP, Kramer GW, Van Schil PE, Legrand C, Smit EF, Schramel F, et al. Randomized controlled trial of resection versus radiotherapy after induction chemotherapy in stage IIIA-N2 non-small-cell lung cancer. J Natl Cancer Inst. 2007;99:442-50 